Dossier: V Simposio Internacional y IX Nacional sobre Derechos de la Niñez y la Adolescencia

\title{
El Programa Aulas de Escucha para estudiantes en riesgo psicosocial
}

\author{
Ana María Jiménez Hidalgo \\ Ministerio de Educación Pública, Costa Rica \\ ana.jimenezhidalgo@ucr.ac.cr \\ https://orcid.org/0000-0002-5966-6988
}

Hellen Carmona Salazar

Consultora UNICEF, Costa Rica

hellenc89@gmail.com

https://orcid.org/0000-0003-3844-0813

Recibido: 1 de julio de 2020

Aceptado: 31 de setiembre de 2020

Resumen: El programa Aulas de Escucha es un modelo de salud mental comunitario para jóvenes en riesgo psicosocial que cursan séptimo año en colegios públicos de las zonas más vulnerables del país. El objetivo de este artículo es presentar la metodología de este modelo, así como los principales resultados e impacto que ha tenido en la población adolescente desde su implementación en el año 2018. Se destaca este espacio como inédito dentro del centro educativo, pues, por medio de tres ejes: clínico (psicológico), creativo y educativo, se brinda a los jóvenes escucha y contención al mismo tiempo que se desarrollan destrezas y habilidades artísticas, deportivas y académicas como posibilidad para sobrellevar su sufrimiento. Los y las docentes a cargo, en conjunto con el estudiantado, participan de un proceso de reflexión constante sobre la violencia y sus fenómenos. Uno de los principales alcances del programa es identificar y acompañar a estudiantes en riesgo de exclusión escolar para lograr su permanencia en el sistema educativo.

\section{(C) $(\Theta \odot$}

La Revista Estudios es editada por la Universidad de Costa Rica y se distribuye bajo una Licencia Creative Commons Atribución-NoComercial-CompartirIgual 3.0 Costa Rica. Para más información envíe un mensaje a 
Dossier: V Simposio Internacional y IX Nacional sobre Derechos de la Niñez y la Adolescencia

Palabras clave: Educación; adolescencia; riesgo psicosocial; salud mental; prevención de la violencia; exclusión escolar

\title{
Aulas de Escucha as a preventive program for school exclusion
}

\begin{abstract}
Aulas de Escucha is a program of communitarian mental health approach for students of seventh grade consider with psychosocial risk of some of the most vulnerable public high schools of the Ministry of Public Education. The purpose of this essay is to present the model's methodology and the main impact discovered in the adolescents since it's implementation in 2018. Aulas de Escucha stand's as a completely new space developed inside the school, that through three axis: clinical (psychological), creative and educative, that offers teenagers listening and containment, and hability of development of artistic, sports and academic skills as a possibility to process their suffering. Teachers incharge of their students participate all together in a process of constant reflexion of the violence and its associated phenomenons. One of the main approaches of the program, is to identify and accompany students in risk of school exclusion with the goal of maintaining its permanence in the education system.
\end{abstract}

Keywords: Education; adolescence; psychosocial risk; mental health; violence; school exclusion.

\section{Presentación:}

De acuerdo al informe anual de resultados presentado por UNICEF Costa Rica (2019) las disparidades sociales y la exclusión escolar son los dos factores que mayoritariamente vulneran a la población infantil y adolescente en el país. Por ello, es la exclusión escolar uno de los agentes de riesgo de atención prioritaria por su asociación con otras problemáticas como el desempleoí, la violencia, las redes delictivas, el narcotráfico, la trata y la explotación sexual. Esto puede conllevar a un latente debilitamiento de los derechos humanos, un aumento en la desigualdad social y una disminución al acceso a oportunidades. A su vez, la población excluida del sistema educativo se encuentra atravesada por una brecha de género importante, en la cual las mujeres se ubican en desventaja respecto a los hombres,

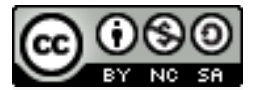

La Revista Estudios es editada por la Universidad de Costa Rica y se distribuye bajo una Licencia Creative Commons Atribución-NoComercial-CompartirIgual 3.0 Costa Rica. Para más información envíe un mensaje a 
Dossier: V Simposio Internacional y IX Nacional sobre Derechos de la Niñez y la Adolescencia

encabezando los índices de pobreza, desempleo, violencia y exclusión escolar (Unicef, 2019).

Es importante reconocer que a pesar de que las cifras de embarazo adolescente y exclusión escolar han disminuidoii, ambas alcanzan en promedio un número alto de 13,000 adolescentes por año (Ministerio de Educación Pública, 2019; UNFPA, 2016). Pero también, aunque los esfuerzos han dado resultados, esta población continúa manteniendo otras condiciones que les amenazan, como el suicidio, que actualmente es la tercer causa de muerte en adolescentes entre $15 \mathrm{y}$ 19 años y la violencia doméstica, que de su porcentaje total el $21 \%$ afecta a niñas y adolescentes (UNICEF, 2019).

De esta forma, comprendemos al igual que Duschatzky y Corea (2009), que los excluidos son en realidad expulsados escolares y representan el resultado de una operación social producida. Son "desaparecidos" del escenario colegial, pierden visibilidad, nombre y palabra, han entrado al universo de la indiferencia "porque transitan por una sociedad que parece no esperar nada de ellos" (Duschatzky y Corea 2009, p.18). Lo consideramos también como el momento culminante de un proceso acumulado de desencuentros del sujeto con su entorno escolar, en donde dicho fracaso es propiamente el de la escuela en cuanto institución encargada de la educación formal, y el de la sociedad en tanto no incorpora a todas y todos sus integrantes de forma plena (Pérez-López y Rodríguez-Arocho, 2017).

Lo anterior ilustra cómo una parte de la población adolescente en Costa Rica es atravesada por diversas dinámicas que la sume en situación de vulnerabilidad, y convierte a sus actores en lo que hemos denominado como jóvenes en riesgo psicosocial, en estas situaciones se conjugan las complejas condiciones sociales de su contexto con las particularidades subjetivas de cada uno y una.

\section{(ब) (ब)}

La Revista Estudios es editada por la Universidad de Costa Rica y se distribuye bajo una Licencia Creative Commons Atribución-NoComercial-CompartirIgual 3.0 Costa Rica. Para más información envíe un mensaje a revistaestudios.eeg@ucr.ac.cr. 
Dossier: V Simposio Internacional y IX Nacional sobre Derechos de la Niñez y la Adolescencia

Cambiar el paradigma desde el cual se lee y aborda a las personas en riesgo de quedar excluidas del sistema educativo es una necesidad ante la urgencia de proponer espacios en los cuales la población adolescente pueda gestionar sus condiciones de vida, adquirir habilidades y herramientas que les permita posicionarse y actuar frente a sus circunstancias.

Este trabajo tiene como objetivo presentar la metodología del modelo Aulas de Escucha, y el impacto de su implementación en veinticuatro centros educativos públicos. La ejecución del programa durante el año 2018 y 2019 es el resultado de mecanismos de coordinación interinstitucional y alianzas público privadas, entre las cuales destacan: la Dirección de Vida Estudiantil (DVE) del Ministerio de Educación Pública (MEP), el Fondo de las Naciones Unidas para la infancia (Unicef), la Fundación Fundamentes, el Hospital Nacional Psiquiátrico Manuel Antonio Chapuí y Torres (HNP) de la Caja Costarricense del Seguro Social (CCSS), el Instituto Costarricense de Drogas (ICD) y la empresa Jiménez y Tanzi, entre otras.

Modelo de salud mental comunitaria en los colegios: Aulas de Escucha

La propuesta de Aulas de Escucha parte del modelo de salud mental comunitario desarrollado por la Fundación Fundamentes y el Hospital Nacional Psiquiátrico desde el año 2007 y sustenta las siguientes premisas:

- "La persona materializa el sufrimiento que generan los contextos de violencia y exclusión.

- La salud mental de las personas y los colectivos tiene que ver con la construcción del vínculo y lazo social.

- La comunidad es el lugar desde donde se puede construir la posibilidad del sostenimiento de lo humano.

- La escucha y la creación son vías de transformación." (Fundamentes, 2018) 
Dossier: V Simposio Internacional y IX Nacional sobre Derechos de la Niñez y la Adolescencia

Este modelo de atención psicosocial se fundamenta en el nudo borromeo utilizado en la topología psicoanalítica para ilustrar la manera en que tres aros se entrelazan de manera interdependiente, no se sabe bien dónde comienza ni dónde termina cada uno. Estas tres bases son la escucha, la creación y el lazo social, los cuales derivan cada uno en los siguientes ejes respectivamente: eje clínico, eje creativo y eje educativo (Fundamentes, 2018).

Aulas de Escucha lleva este modelo de salud mental directamente a las aulas de los colegios más vulnerables de nuestro país, seleccionando a la población que, de acuerdo a las estadísticas del MEP, posee los mayores índices de exclusión escolar, es decir, estudiantes de sétimo añoiii. Con ellos y ellas se trabaja en la transición de la escuela al colegio y de esta forma el programa les brinda un espacio de acuerdo a cada uno de los tres ejes:

1. Eje clínico - emocional: Por medio de un taller de habilidades para la vida se fortalecen herramientas en favor del desarrollo personal y social a partir de un proceso grupal de contención y acompañamiento emocional. Dicho taller se estructura de acuerdo a módulos que buscan cubrir las principales problemáticas tales como prevención de la violencia, el fenómeno de las drogas y temas en materia de derechos en salud sexual, reproductiva, diversidades y proyecto de vida. Estos talleres los imparten profesionales en psicología, que además habilitan espacios de escucha individual de acuerdo con la necesidad del estudiantado.

2. Eje creativo: Se favorece el potencial creativo, artístico, plástico, escénico y deportivo mediante el arte y recreación física. Se desarrolla por medio de un taller de escultura y dibujo a cargo de docentes de artes plásticas y un taller de judo impartido por la Federación Costarricense de Judo.

\footnotetext{
(c) (1)(2)

La Revista Estudios es editada por la Universidad de Costa Rica y se distribuye bajo una Licencia Creative Commons Atribución-NoComercial-CompartirIgual 3.0 Costa Rica. Para más información envíe un mensaje a revistaestudios.eeg@ucr.ac.cr.
} 
Dossier: V Simposio Internacional y IX Nacional sobre Derechos de la Niñez y la Adolescencia

3. Eje educativo: Se brinda apoyo pedagógico con tutorías de recuperación. Estas son impartidas por docentes del centro educativo, a quienes se les asignan lecciones adicionales para atender a la población estudiantil. Están enfocadas en el desarrollo de habilidades de aprendizaje, las cuales se proponen generalmente a partir de actividades lúdicas, repaso de contenidos y apoyo en las actividades extracurriculares.

Estos talleres funcionan como sitios de protección y consolidan al centro educativo como un espacio seguro y atractivo para las personas adolescentes. Facilita el acceso a actividades especiales dentro de la institución que involucran la música, el teatro, el baile, el deporte y los medios de comunicación.

Cobertura y alcance

El plan piloto que inicia en el 2018 cubre veinte colegios identificados por la Dirección de Vida Estudiantil del MEP como prioritarios para el abordaje de la prevención de la violencia. El siguiente mapa muestra las zonas de atención:

\section{(c) (i) (9)}

La Revista Estudios es editada por la Universidad de Costa Rica y se distribuye bajo una Licencia Creative Commons Atribución-NoComercial-CompartirIgual 3.0 Costa Rica. Para más información envíe un mensaje a revistaestudios.eeg@ucr.ac.cr. 
Dossier: V Simposio Internacional y IX Nacional sobre Derechos de la Niñez y la Adolescencia

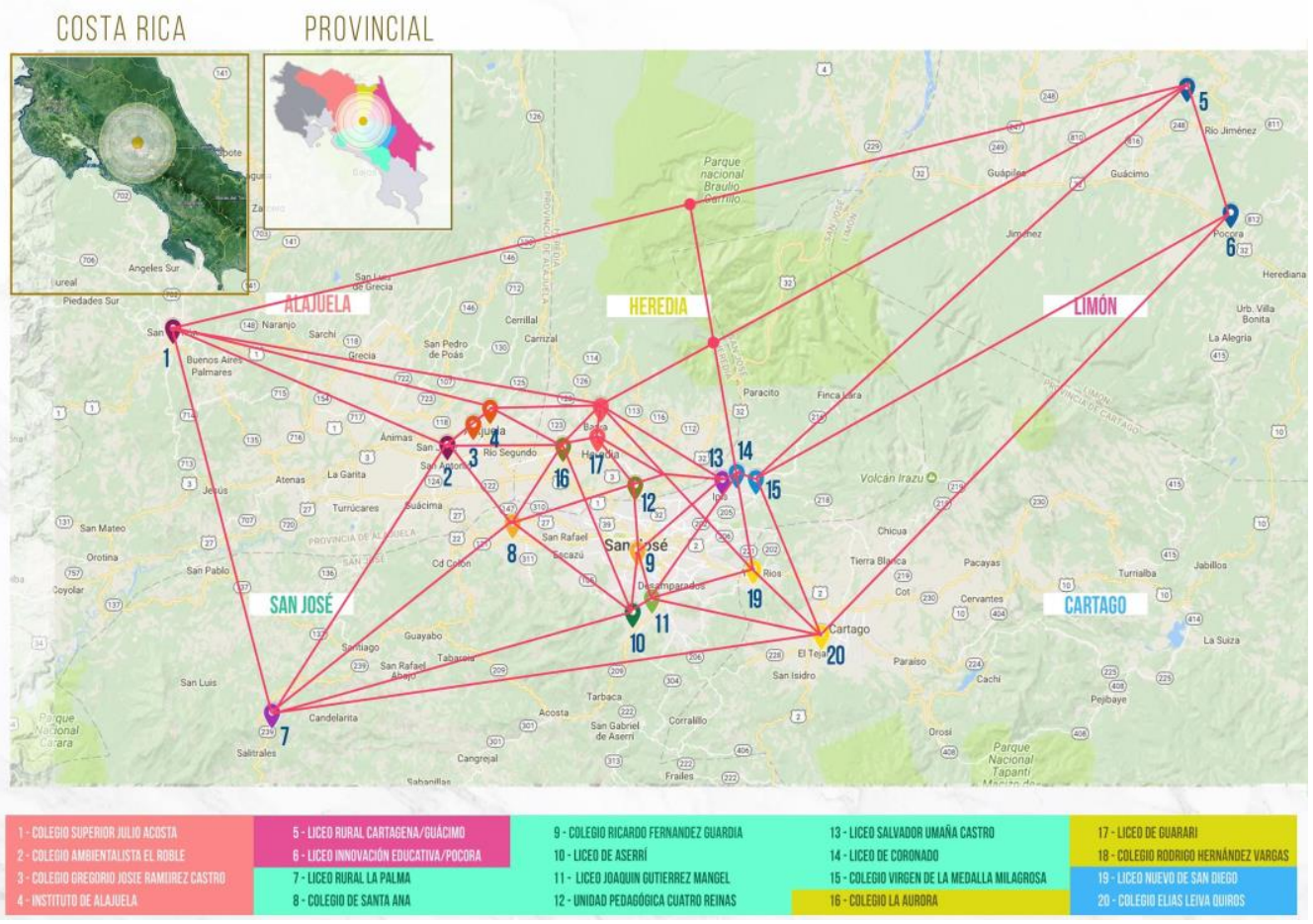

Figura 1: Mapa con la ubicación de los 20 colegios de Aulas de Escucha del 2018. Elaboración propia.

En el 2018, durante los meses de abril a agostoํㅡ, se logró atender a 1029 estudiantes de sétimo año inscritos en el programa, cuya distribución se puede observar en el siguiente gráfico:

\section{(c) (i) (2) (2)}

La Revista Estudios es editada por la Universidad de Costa Rica y se distribuye bajo una Licencia Creative Commons Atribución-NoComercial-CompartirIgual 3.0 Costa Rica. Para más información envíe un mensaje a revistaestudios.eeg@ucr.ac.cr. 
Dossier: V Simposio Internacional y IX Nacional sobre Derechos de la Niñez y la Adolescencia

\section{Total del matrícula y Promedio de asistencia por CE}

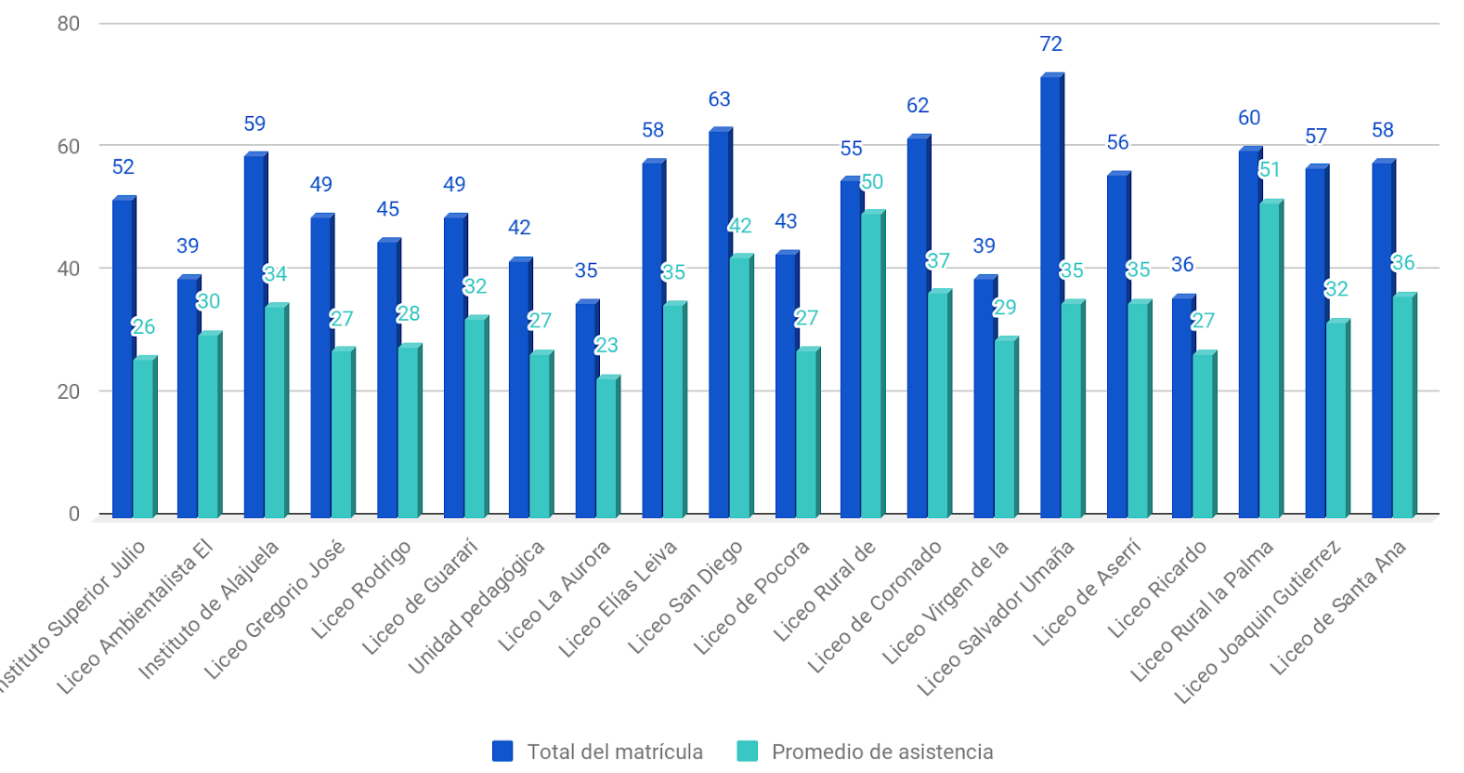

Figura 2: Gráfico que representa en azul la cantidad de estudiantes inscritos y en turquesa el promedio de asistencia por cada centro educativo para el 2018. Elaboración propia. $^{v}$

\section{(c) (1) (2)}

La Revista Estudios es editada por la Universidad de Costa Rica y se distribuye bajo una Licencia Creative Commons Atribución-NoComercial-CompartirIgual 3.0 Costa Rica. Para más información envíe un mensaje a revistaestudios.eeg@ucr.ac.cr. 
Dossier: V Simposio Internacional y IX Nacional sobre Derechos de la Niñez y la Adolescencia

Para el 2019 el programa se reestructura de acuerdo a cuatro regiones, cada una con seis colegios, presentadas en la siguiente tabla:

\begin{tabular}{|c|c|c|c|c|}
\hline \multicolumn{3}{|c|}{ Región 1: GAM } & \multicolumn{2}{|c|}{ Región 2: Alajuela } \\
\hline Region & $\begin{array}{l}\text { Dirección } \\
\text { al del MEP }\end{array}$ & Colegio & $\begin{array}{c}\text { Dirección } \\
\text { Regional del MEP }\end{array}$ & Colegio \\
\hline Oeste & San José & $\begin{array}{l}\quad \text { Unidad } \\
\text { Pedagógica Cuatro } \\
\text { Reinas }\end{array}$ & Alajuela & $\begin{array}{l}\text { Instituto de } \\
\text { Alajuela }\end{array}$ \\
\hline Norte & San José & $\begin{array}{l}\text { Liceo de } \\
\text { Coronado }\end{array}$ & Alajuela & \begin{tabular}{l}
\multicolumn{2}{c}{ Liceo } \\
Gregorio \\
Ramírez
\end{tabular} \\
\hline Oeste & San José & $\begin{array}{l}\text { Liceo } \\
\text { Ricardo Fernández } \\
\text { Guardia }\end{array}$ & Occidente & \begin{tabular}{l}
\multicolumn{2}{c}{ Instituto } \\
Superior Julio \\
Acosta García
\end{tabular} \\
\hline & Cartago & $\begin{array}{l}\text { Liceo Nuevo } \\
\text { de San Diego }\end{array}$ & Occidente & \begin{tabular}{l}
\multicolumn{1}{c}{ Liceo } \\
Nuestra Señora de \\
los Ángeles
\end{tabular} \\
\hline dos & Desampara & Aserrí ${ }^{\text {Liceo de }}$ & Occidente & $\begin{array}{l}\text { Colegio de } \\
\text { Naranjo }\end{array}$ \\
\hline & Heredia & $\underset{\text { Guarari }}{\text { Liceo de }}$ & San Carlos & Carlos $^{\text {Liceo San }}$ \\
\hline
\end{tabular}

La Revista Estudios es editada por la Universidad de Costa Rica y se distribuye bajo una Licencia Creative Commons Atribución-NoComercial-CompartirIgual 3.0 Costa Rica. Para más información envíe un mensaje a 
Dossier: V Simposio Internacional y IX Nacional sobre Derechos de la Niñez y la Adolescencia

\begin{tabular}{|c|c|c|c|}
\hline \multicolumn{2}{|c|}{ Región 2: Caribe } & \multicolumn{2}{|c|}{ Región 3: Pacífico } \\
\hline $\begin{array}{c}\text { Dirección } \\
\text { Regional del MEP }\end{array}$ & Colegio & $\begin{array}{c}\text { Dirección } \\
\text { Regional del MEP }\end{array}$ & Colegio \\
\hline Limón & $\begin{array}{l}\text { Liceo Nuevo } \\
\text { de Limón Mario } \\
\text { Bourne Bourne }\end{array}$ & Puntarenas & $\begin{array}{l}\text { Liceo de } \\
\text { Chacarita }\end{array}$ \\
\hline Limón & $\begin{array}{ll}\text { Liceo Río } \\
\text { Banano }\end{array}$ & Puntarenas & $\begin{array}{l}\text { Liceo de } \\
\text { Miramar }\end{array}$ \\
\hline Guápiles & Cariari ${ }^{\text {Liceo }}$ de & Puntarenas & $\begin{array}{l}\text { Liceo diurno } \\
\text { José Martí }\end{array}$ \\
\hline Guápiles & $\begin{array}{l}\text { Liceo de } \\
\text { Pocora }\end{array}$ & Puntarenas & $\begin{array}{c}\text { Liceo } \\
\text { Obando Chan }\end{array}$ \\
\hline Sarapiquí & Virgen ${ }^{\text {Liceo la }}$ & Puntarenas & $\begin{array}{l}\text { Liceo } \\
\text { Esparza }\end{array}$ \\
\hline Sarapiquí & Frío $\quad$ Liceo Río & Aguirre & $\begin{array}{c}\text { Liceo } \\
\text { Quebrada Ganado }\end{array}$ \\
\hline
\end{tabular}

Figura 3: Distribución de colegios de Aulas de Escucha por región para el 2019. Elaboración propia.

La Revista Estudios es editada por la Universidad de Costa Rica y se distribuye bajo una Licencia Creative Commons Atribución-NoComercial-CompartirIgual 3.0 Costa Rica. Para más información envíe un mensaje a revistaestudios.eeg@ucr.ac.cr. 
Dossier: V Simposio Internacional y IX Nacional sobre Derechos de la Niñez y la Adolescencia

Para este año 2019 la cobertura al corte de agosto es de 1267 estudiantes matriculados, cuya distribución regional se muestra en el siguiente gráfico:

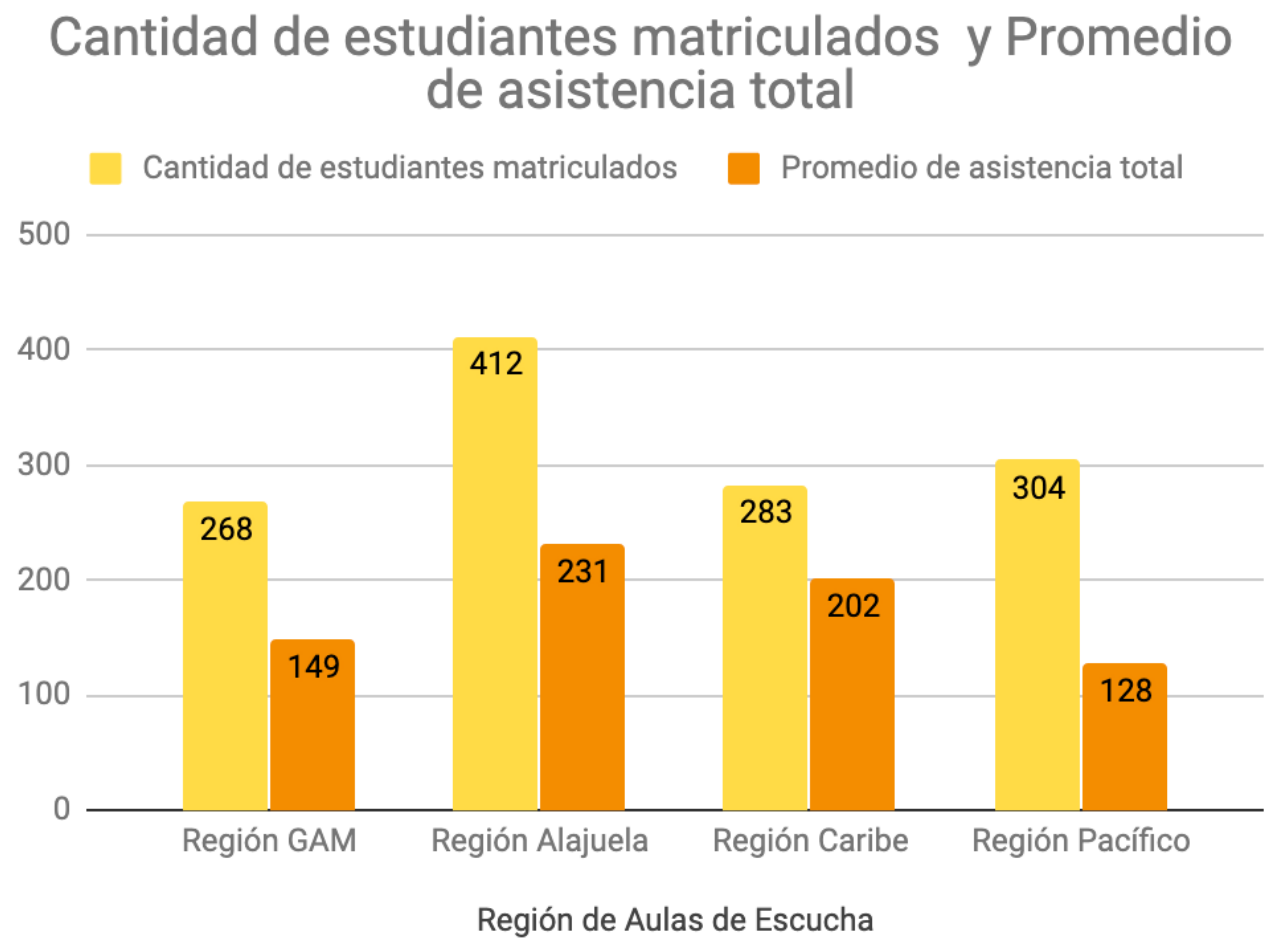

Figura 4: Gráfico con la distribución de estudiantes participantes por región al 2019. Elaboración propia.

La barra amarilla indica la cantidad de estudiantes matriculados en el proyecto y la naranja, por su parte, da cuenta de la cantidad de estudiantes que asisten regularmente. La diferencia que indican ambas barras representa un ausentismo de participantes que responde a situaciones como: atraso en los nombramientos de facilitadores, cambios de docentes, ausencias debido a días feriados, congresos de sindicatos, semanas de exámenes, consejos de profesores, actividades especiales como celebraciones en los centros educativos así como problemas de comunicación directa con el estudiantado a la hora de convocarlos a

\section{(c) (i) (2) (2)}

La Revista Estudios es editada por la Universidad de Costa Rica y se distribuye bajo una Licencia Creative Commons Atribución-NoComercial-CompartirIgual 3.0 Costa Rica. Para más información envíe un mensaje a revistaestudios.eeg@ucr.ac.cr. 
Dossier: V Simposio Internacional y IX Nacional sobre Derechos de la Niñez y la Adolescencia los talleres. Solventar estas dificultades es parte de los retos del programa. En cuanto a cantidad de sesiones realizadas, de abril a agosto del 2019 se han desarrollado en promedio veinte por colegio. Esta proporción está determinada por factores vinculados a la agilidad de los nombramientos a cargo del departamento de recursos humanos del MEP. La sumatoria de las sesiones por cada región se muestran en el siguiente gráfico:

\section{Cantidad de sesiones realizadas en promedio}

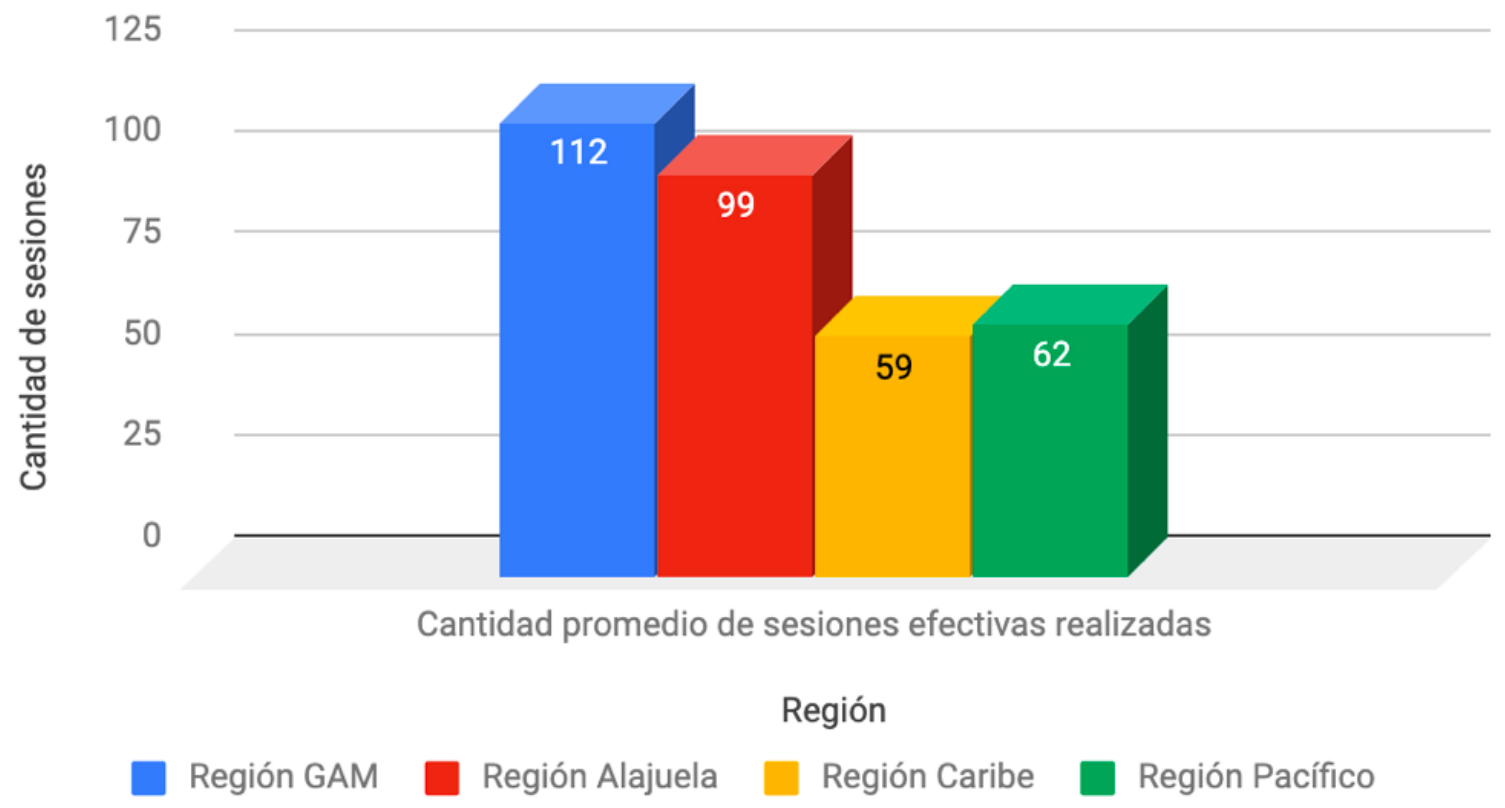

Las regiones con más sesiones iniciaron primero y son las que se concentran en el área metropolitana donde se cuenta con mayor recurso humano a diferencia de Limón y Puntarenas. Se espera para el 2020 aumentar la cobertura y el alcance del proyecto al incorporar la región de Guanacaste, y posteriormente, cada año cubrir nuevas regiones. Finalmente, la meta es abarcar aquellas zonas y colegios donde se concentran la mayor parte de estudiantes vulnerables a la exclusión escolar. Otro esfuerzos se asocian a darle continuidad a estos espacios con

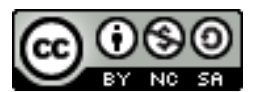

La Revista Estudios es editada por la Universidad de Costa Rica y se distribuye bajo una Licencia Creative Commons Atribución-NoComercial-CompartirIgual 3.0 Costa Rica. Para más información envíe un mensaje a revistaestudios.eeg@ucr.ac.cr. 
Dossier: V Simposio Internacional y IX Nacional sobre Derechos de la Niñez y la Adolescencia estudiantes de octavo año, con el fin de intentar responder a una demanda que ellos y ellas mismas nos han realizado.

\section{Un espacio inédito: el impacto de Aulas de Escucha en el estudiantado}

Aulas de Escucha es un espacio inédito para los jóvenes porque se sienten comprendidos y escuchados a la vez que desarrollan destrezas y habilidades artísticas, deportivas y académicas. Esto determina el logro del espacio, en el cual la población adolescente, por medio de la escucha, encuentra la posibilidad de gestionar sus sentimientos, miedos, temores, angustias, situaciones de conflicto así como alegrías, esperanzas e ilusiones. Simultáneamente, docentes en conjunto con la población adolescente participan de un proceso de reflexión constante sobre la violencia y sus fenómenos. Lo anterior permite un cambio en el imaginario de esta población, dejan de ser identificados como problemáticos para pasar a representar un conjunto positivo dentro de la dinámica del colegio, es decir, pasan de la etiqueta al deseo, del estigma al valor. Esto implica para cada uno/a la conciencia de su aporte a la sociedad y su capacidad creativa junto con el deseo de permanecer en el colegio.

Aulas de Escucha también representa un espacio transicional que brinda un acompañamiento en el pasaje de la infancia a la adolescencia, esto es, de la escuela al colegio. Brinda la contención que el estudiantado requiere frente a situaciones de violencia diarias en su familia, su barrio y en el centro educativo. Esto permite cuestionar las problemáticas que comúnmente se han asociado a la adolescencia y verles como situaciones propias de su contexto, para darles un nuevo significado desde el crecimiento y la posibilidad de creación. Este cuestionamiento al imaginario negativo construido alrededor de la adolescencia permite posicionar al adolescente frente a sus condiciones y su contexto, generando un reconocimiento de la violencia como algo que está naturalizado por su experiencia inmediata, pero que no

\section{(c) (i) (9) (2)}

La Revista Estudios es editada por la Universidad de Costa Rica y se distribuye bajo una Licencia Creative Commons Atribución-NoComercial-CompartirIgual 3.0 Costa Rica. Para más información envíe un mensaje a 
Dossier: V Simposio Internacional y IX Nacional sobre Derechos de la Niñez y la Adolescencia necesariamente debe ser parte de su futuro y de su proyecto de vida. Esto rompe con su lógica e interrumpe la dinámica de víctima y victimario, lo que permite la construcción de vínculos mediados por la empatía y el acompañamiento.

La ruptura con la dinámica de violencia y sus manifestaciones ponen en marcha reflexiones a los jóvenes que les permite deconstruir su lugar frente a la violencia sexual y de género. Esto mediante un proceso de sensibilización sobre los roles y estereotipos, sobre cómo se construyen los vínculos que median por la desigualdad entre hombres y mujeres. Esto es la antesala para propiciar discusiones en torno al diálogo y a la construcción de nuevas formas de vincularse sin importar el tipo de relación (noviazgo, amistad, compañerismo, familia, entre otros). En este proyecto, sus participantes han sido capaces de reconocer y cuestionar algunas manifestaciones de la violencia de género como el acoso callejero, las relaciones impropias y las masculinidades tóxicas.

Lo anterior también ha permitido atribuir otro significado a la corporalidad y al imaginario del cuerpo, el cual deja de ser depositario de la violencia en muchas de sus formas, para convertirse en un lugar del cuidado de sí mismo. Esto se asocia a que la población adolescente refiere sufrir su corporalidad desde la construcción del ideal de belleza hasta situaciones de violencia sexual, pasando por el abuso físico, encontramos que en muchos casos la respuesta a este sufrimiento son las autolesiones. Ante estos casos, el espacio de Aulas de Escucha ofrece la vía de la palabra, el arte y la creación para que sus cuerpos no continúen estableciéndose como lienzos del dolor.

El trabajo diario en estos temas que se realiza por medio de los talleres corresponden a los principales resultados que el programa tiene en la población y que contribuyen indirectamente a prevenir la exclusión escolar. Sin embargo, mediante este espacio es posible identificar a aquellos/as estudiantes que no 
Dossier: V Simposio Internacional y IX Nacional sobre Derechos de la Niñez y la Adolescencia pueden sostener más su permanencia en el centro educativo. Con estos y estas se inicia un proceso de seguimiento y acompañamiento singular, que incluye la atención psicológica y el compromiso institucional por medio del departamento de orientación, quienes se encargan de coordinar e integrar al equipo de Aulas de Escucha a reuniones con los padres, madres de familia, personas cuidadoras del menor y redes de apoyo institucional. Con esta estrategia se logró que en el 2018 ningún estudiante participante del proyecto abandonara el sistema educativo.

Por último, un objetivo importante del programa es lograr que las y los adolescentes se reconozcan a sí mismos como sujetos de derecho y personas responsables de sus actos, que merecen convivir en espacios libres de violencia en los cuales se les respete y dignifique. De esta forma el centro educativo pasa de ser un lugar de exclusión a ser como un espacio seguro.

\section{Conclusiones:}

En miras de la apuesta a un desarrollo sostenible de nuestro país, es responsabilidad del Estado garantizar el cumplimiento y la promoción de los derechos de los y las adolescentes. Sin embargo, se ha encontrado que para instituciones como el MEP esto no es posible sin cooperación que realiza con otras entidades estatales, organizaciones del tercer sector y las Naciones Unidas, quienes conscientes de la situación de la población adolescente en nuestro país, han logrado por medio de Aulas de Escucha crear y consolidar espacios en los cuales las subjetividades sean el tema de discusión. Lo anterior se ha dado mediante un abordaje de las necesidades particulares de las comunidades educativas y de la población con la cual se trabaja.

La propuesta metodológica de este proyecto y su recurso humano se colocan como el punto fuerte del proyecto, principalmente por el abordaje integral e interdisciplinario que implica el desarrollo de los talleres. Para las y los facilitadores 
Dossier: V Simposio Internacional y IX Nacional sobre Derechos de la Niñez y la Adolescencia cada uno de los jóvenes con quienes trabajan dejan de ser un número más en las listas y se convierten en personas con historias de vida, familias, dificultades, pero que al mismo tiempo cuentan con muchas ganas de ser tomados en cuenta, de ser valorados y de tener una voz.

Por ello, cobra sentido que la principal herramienta de trabajo e investigación de este plan sea la escucha a la persona adolescente, lo cual representa también un acercamiento a sus realidades, sentires[cambiar término, se sugiere "apreciaciones" o "sentimientos"] y necesidades, y as,í se puedan potenciar los alcances en materia de derechos de las personas adolescentes.

Finalmente, el programa, así como disfruta de los buenos resultados también cada día enfrenta una serie de retos y obstáculos que van desde la inestabilidad de sus financiamientos externos, hasta la falta de apoyo administrativo o de espacios físicos dentro de algunos colegios. De esta forma, lejos de estar acabado, este proyecto es un ente vivo que debe adaptarse constantemente a las circunstancias de su entorno, diversas en cada región, y que en ocasiones encuentra resistencias para potenciar la cultura de cambio y desarrollo que desea.

\section{Bibliografía:}

Duschatzky, S y Corea, C. (2009). Chicos en banda: los caminos de la subjetividad en el declive de las instituciones. Buenos Aires: Paidós.

Fundamentes. (2018). Sistematización: Programa Preventivo Casitas de Escucha. Limón y Talamanca. Fundamentes y UNICEF.

Ministerio de Educación Pública. (2019). Estrategia del MEP logró reducir la exclusión escolar pese a huelga. Recuperado de: https://www.mep.go.cr/noticias/estrategia-mep-logro-reducir-exclusionescolar-pese-huelga-0

\section{(c) (1) (2)}

La Revista Estudios es editada por la Universidad de Costa Rica y se distribuye bajo una Licencia Creative Commons Atribución-NoComercial-CompartirIgual 3.0 Costa Rica. Para más información envíe un mensaje a revistaestudios.eeg@ucr.ac.cr. 
Dossier: V Simposio Internacional y IX Nacional sobre Derechos de la Niñez y la Adolescencia

Ministerio de Educación Pública. (2019). Exclusión en colegios 2014-2018. Recuperado de: https://www.mep.go.cr/indicadores edu/autotabulaciones.html

Pérez-López, S \& Rodríguez-Arocho, W. (2017). Una mirada crítica al fracaso escolar. Pedagogía, $49 . \quad$ Recuperado de http://revistapedagogia.uprrp.edu/?p=1058

UNICEF. (2019). Informe Anual de Resultados 2018. Recuperado de: https://unicefmy.sharepoint.com/personal/imadrigal unicef org1/ layouts/15/Doc.aspx?s ourcedoc=\%7B4c09b115-19ec-4d20-a740fc85b0eaba09\%7D\&action=default\&slrid=f644fa9e-00c9-9000-253b2a988ae7dd61\&originalPath=aHR0cHM6Ly91bmliZWYtbXkuc2hhcmVwb2I udC5jb20vOnA6L2cvcGVyc29uYWwvam1hZHJpZ2FsX3VuaWNIZI9vcmcxL OVSV3hDVXpzR1NCTnAwRDhoYkRxdWdrQIBzLXZKR1JvbnpDUU5YTEd0 UFd0Y1E cnRpbWU9UW9vYIInSWgxMGc\&cid=1624d38a-71d2-4453$\underline{9743-a 823 d 2 b 80891}$

UNFPA. (2016). Demasiado joven para ser mamá. Recuperado de: https://lac.unfpa.org/es/noticias/demasiado-joven-para-ser-mam\%C3\%A1

\section{Notas}

\footnotetext{
${ }^{\mathrm{i}}$ De acuerdo al Informe Anual de Resultados Unicef Costa Rica 2019, en nuestro país hay 175,328 jóvenes entre los 15 y los 24 años que no estudian ni trabajan, de los cuales 103,246 son mujeres, un 58\%.

${ }^{\text {ii }}$ El porcentaje de embarazo adolescente pasó del $19.4 \%$ en el 2012 a $14.7 \%$ en el 2017, mientras que la exclusión escolar en III ciclo pasó de ser 7,2\% en el 2017 a un 3,5\% en el año anterior.

iii Para el 2018 la exclusión escolar en sétimo representó el 34,9\% del total en secundaria, esto según datos de la Estadística sobre Exclusión Escolar del Ministerio de Educación Pública.

${ }^{\text {iv }}$ Debido a la huelga del sector educativo, la mayoría de los colegios no funcionó regularmente.

${ }^{\vee}$ En la siguiente figura se visualizan los nombres completos de los colegios.
}

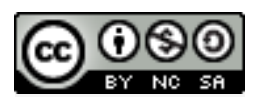

La Revista Estudios es editada por la Universidad de Costa Rica y se distribuye bajo una Licencia Creative Commons Atribución-NoComercial-CompartirIgual 3.0 Costa Rica. Para más información envíe un mensaje a revistaestudios.eeg@ucr.ac.cr. 